\title{
Health-related quality of life in patients with locally advanced or metastatic breast cancer treated with eribulin mesylate or capecitabine in an open-label randomized phase 3 trial
}

\author{
Javier Cortes ${ }^{1,2} \cdot$ Stacie Hudgens $^{3} \cdot$ Chris Twelves $^{4} \cdot$ Edith A. Perez $^{5}$. \\ Ahmad Awada ${ }^{6}$. Louise Yelle ${ }^{7}$ Susan McCutcheon ${ }^{8}$ - Peter A. Kaufman' \\ Anna Forsythe $^{10} \cdot$ Galina Velikova ${ }^{4}$
}

Received: 29 July 2015/Accepted: 3 November 2015/Published online: 14 November 2015

(c) The Author(s) 2015. This article is published with open access at Springerlink.com

\begin{abstract}
The clinical benefit of eribulin versus capecitabine was evaluated using health-related quality of life (HRQoL) data from a phase 3 randomized trial in patients with pretreated advanced/metastatic breast cancer (ClinicalTrials.gov identifier: NCT00337103). The study population has been described previously (Kaufman et al. in J Clin Oncol 33:594-601, 2015). Eligible patients received eribulin $\left(1.4 \mathrm{mg} / \mathrm{m}^{2}\right.$ intravenously on days 1 and 8$)$ or capecitabine $\left(1.25 \mathrm{~g} / \mathrm{m}^{2}\right.$ orally twice daily on days $\left.1-14\right)$ per 21-day cycles. HRQoL was assessed using the European Organisation for Research and Treatment of Cancer (EORTC) Quality-of-life Questionnaire-Core 30 questions (QLQ-C30) and breast module-23 questions (QLQ-BR23), administered at baseline through 24 months, until disease progression or other antitumor treatment initiation. Minimally important difference (MID) and time to symptom
\end{abstract}

Electronic supplementary material The online version of this article (doi:10.1007/s10549-015-3633-7) contains supplementary material, which is available to authorized users.

Galina Velikova

g.velikova@leeds.ac.uk

1 Vall d'Hebron Institute of Oncology (VHIO), Barcelona, Spain

2 Oncology Department, Ramon y Cajal University Hospital, Madrid, Spain

3 Department of Quantitative Science, Clinical Outcomes Solutions, 3709 North Campbell, Tucson, AZ, USA

4 Department of Oncology, Leeds Institute of Cancer and Pathology, and St James's Institute of Oncology, University of Leeds, Leeds LS9 7TF, England, UK

5 Division of Hematology/Oncology, Department of Cancer Biology, Mayo Clinic, Jacksonville, FL, USA worsening (TSW) were investigated. 1062 (96.4\%) Patients completed the EORTC questionnaire at baseline; overall, compliance was $\geq 80 \%$. Patients receiving capecitabine versus eribulin had significantly worse symptoms (higher scores) for nausea/vomiting (MID 8; $P<0.05$ ) and diarrhea (MID 7; $P<0.05$ ). Treatment with eribulin versus capecitabine, led to worse systemic therapy side-effects (dry mouth, different tastes, irritated eyes, feeling ill, hot flushes, headaches, and hair loss; MID 10; $P<0.01$ ). Clinically meaningful worsening was observed for future perspective (MID 10; $P<0.05$ ) with capecitabine and for systemic therapy side-effects scale (MID 10; $P<0.01$ ) with eribulin. Patients receiving capecitabine experienced more-rapid deterioration in body image (by 2.9 months) and future perspective (by 1.4 months; $P<0.05$ ) compared with those on eribulin; the opposite was observed for systemic side-effects where patients receiving eribulin experienced more-rapid deterioration than those receiving capecitabine (by 2 months; $P<0.05$ ). Eribulin and

6 Medical Oncology Clinic, Medicine Department, Institut Jules Bordet, Université Libre de Bruxelles, Brussels, Belgium

7 Department of Medicine, University of Montreal, Montreal, QC, Canada

8 Formerly of Eisai Ltd, Hatfield, England, UK

9 Section of Hematology/Oncology, Norris Cotton Cancer Center, Dartmouth-Hitchcock Medical Center, Lebanon, NH, USA

10 Department of Global Value and Access Strategy, Eisai Inc., Woodcliff Lake, NJ, USA 
capecitabine were found to have similar impact on patient functioning with no overall difference in HRQoL. Patients receiving eribulin reported worse systemic side-effects of chemotherapy but reduced gastrointestinal toxicity compared with capecitabine.

Keywords Breast cancer - Eribulin - Quality of life . Minimally important difference $\cdot$ Side effects

\section{Introduction}

Despite recent advances in cancer therapies and increased availability of treatment options, metastatic breast cancer (MBC) remains incurable. The main treatment goals are to achieve disease control, preferably through prolonging overall survival (OS), and to delay or prevent debilitating disease symptoms [1, 2]. With each additional line of therapy, the response to chemotherapy decreases further and the response rate may be as low as $15 \%$ in patients who have received up to two prior therapies, and is associated with significant toxicity and relatively low OS [3, 4]. In this situation, the measurement of patient-reported symptom experience and health-related quality of life (HRQoL) can provide additional information to evaluate and compare the efficacy and toxicity profiles of the treatments. Further, incorporation of patient-reported outcomes into toxicity reporting in clinical trials has been recommended to overcome the potential underreporting of severity of subjective adverse events by physicians in clinical trials [5-7].

Treatment side-effects (even those of lower grade) may adversely impact patient well-being [8, 9]. For example, chemotherapy-induced gastrointestinal symptoms (nausea, vomiting, and diarrhea) are not life-threatening, but are associated with worse HRQoL [10]. In contrast, uncomplicated neutropenia is often not associated with significant symptoms.

Eribulin mesylate (eribulin), a novel microtubule dynamics inhibitor, was the first single agent shown to improve survival in patients with heavily pretreated $\mathrm{MBC}$ in the phase 3, randomized study 305/EMBRACE trial, where patients receiving eribulin experienced 2.7 months longer median OS than those receiving treatment of physician's choice (hazard ratio (HR) 0.81 ; $95 \%$ confidence interval (CI) $0.67-0.96 ; P=0.014$ ) [11]. HRQoL was not assessed in the trial due to the variety of treatments and schedules in the control arm (treatment of physician's choice).

HRQoL was, however, a prespecified secondary endpoint in a second phase 3, open-label, randomized trial (study 301) that evaluated eribulin versus capecitabine as first- to third-line treatment in pretreated patients with
MBC [12]. The differences observed in OS between the eribulin arm compared with capecitabine (15.9 vs. 14.5 months, respectively) were not statistically significant (HR 0.88; $95 \%$ CI 0.77-1.00; $P=0.056$ ). Overall, the safety and tolerability profiles of the treatments were comparable: nausea was common with both treatments, in addition, eribulin treatment more commonly led to neutropenia, alopecia, leukopenia, and global peripheral neuropathy; whereas capecitabine was more often associated with hand-foot syndrome, and diarrhea. Similar improvements in patients' HRQoL over time (a prespecified secondary endpoint), measured by the global health status (GHS)/quality of life (QoL) subscale of the European Organisation for Research and Treatment of Cancer Quality-of-life Questionnaire-Core 30 questions (EORTC QLQ-C30), were observed in both treatment arms [12].

These two trials led to the approval of eribulin as a monotherapy for patients with $\mathrm{MBC}$ who have previously received at least one (European Union) or two (United States) chemotherapeutic regimens for advanced/metastatic disease, where prior therapy included an anthracycline and a taxane in the adjuvant or metastatic setting $[13,14]$.

Here we compare and further evaluate the clinical impact of eribulin and capecitabine on patients' symptoms/ side-effects, functioning, and HRQoL in study 301 [12] to better understand the quality of survival in patients with MBC. The analysis and interpretation of the results are based on a model that posits biological factors associated with a disease or its treatment lead to symptoms that influence functional status, which then impacts on overall HRQoL [15]. The specific objectives of the current post hoc analyses were to:

(a) Compare physical symptoms, functional scores, and GHS/QoL in patients treated with eribulin versus capecitabine over time;

(b) Estimate the proportion of patients experiencing clinically meaningful changes in HRQoL scales; and

(c) Compare the time to meaningful deterioration of HRQoL in both treatment arms.

Subgroup analyses in patients with human epidermal growth factor receptor 2 (HER2)-negative and triple-negative disease status were also performed.

\section{Methods}

\section{Patients}

The population enrolled (E7389-G000-301; ClinicalTrials.gov identifier: NCT00337103) has been previously described [12]. In brief, women (aged $\geq 18$ years) with 
histologically or cytologically confirmed breast cancer, who had received $\leq 3$ prior chemotherapy regimens ( $\leq 2$ for advanced and/or metastatic disease) including prior therapy with an anthracycline and a taxane, were eligible for study inclusion.

\section{Study design}

The study was an open-label, 2-arm, parallel, multicenter, phase 3 trial in which patients were stratified at randomization by geographic region (North America, Western Europe, Eastern Europe, Latin America, South Africa, and Asia) and HER2 status (positive, negative, or unknown). Patients were randomized $(1: 1)$ to receive 21 -day cycles comprising eribulin mesylate $1.4 \mathrm{mg} / \mathrm{m}^{2}$ (equivalent to $1.23 \mathrm{mg} / \mathrm{m}^{2}$ of eribulin expressed as free base) intravenously over $2-5 \mathrm{~min}$ on days 1 and 8 , or capecitabine $1.25 \mathrm{~g} / \mathrm{m}^{2}$ orally twice daily on days $1-14$. Patients received study treatment until disease progression, unacceptable toxicity, or patient/investigator request to discontinue. Grade 3 and 4 toxicities, including certain grade 2 toxicities with capecitabine, were managed by dose modification including dose reduction, treatment interruption, and/or symptomatic treatment [12].

\section{HRQoL assessment}

HRQoL was a secondary endpoint in this study; the principal prespecified HRQoL outcome was overall GHS/QoL at week 6, and has been reported previously in brief [12]. The results reported here are based on additional post hoc analyses of the study data.

HRQoL was assessed using EORTC QLQ-C30 (version 3.0) $[16,17]$ and the breast module-23 questions (QLQBR23; version 1.0) [18]. The QLQ-C30 consists of 30 questions addressing five functional scales (cognitive, emotional, physical, social, and role), nine symptom scales (appetite loss, constipation, diarrhea, dyspnea, fatigue, financial difficulties, insomnia, nausea and vomiting, and pain), and one GHS/QoL scale. The EORTC QLQ-BR23 focuses on breast-cancer-specific issues and includes 23 questions addressing four functional (body image, future perspective, sexual enjoyment, and sexual functioning) and symptom scales (arm symptoms, breast symptoms, systemic therapy side-effects, and upset by hair loss) [19]. All scores for the EORTC QLQ-C30 and EORTC QLQ-BR23 were transformed to a scale from 0 to 100 [19]. Higher scores in the functional scales and GHS/QoL represent a superior level of functioning and better HRQoL, whereas higher scores in the symptom scales or items represent worse symptoms.

The questionnaires were administered at baseline, week 6 , and months $3,6,12,18$, and 24 , or until disease progression or initiation of other antitumor treatment (including those initiated after study termination). The baseline EORTC questionnaires were completed in clinic before randomization. Subsequent questionnaires were completed in the clinic before any study-related procedures for that visit and before tumor assessment results were communicated to the patient. Patients were asked to complete questionnaires at each clinic visit, even if they had declined previously. Compliance was assessed by counting completed questionnaires.

\section{Statistical analyses}

The HRQoL population was defined as patients with QoL assessments at each time point within the intent-to-treat (ITT) population. Data were also analyzed separately for patients with HER2-negative or triple-negative disease. Analysis of patients with HER2-positive disease were not planned due to the anticipated fewer number of patients in this subgroup.

Compliance for completing the EORTC questionnaires was evaluated descriptively for each treatment group. Pattern-mixture models were used to account for data missing-not-at-random [20]. No imputation for missing data was conducted. Mixed models on a set of covariates based on expert opinion (baseline patient demographics such as age, HER2 status, hormone receptor status, Eastern Cooperative Oncology Group status, number of prior chemotherapy regimens for advanced disease, number of organs involved, visceral involvement, and disease-free interval $>1$ year prior to study) were performed to estimate the effect difference on repeated responses over a selected period of time and between treatment arms. Longitudinal analysis outcomes were expressed as least squares mean and standard error. To test the difference in least squares mean change from baseline between treatment arms, a 2-sided test with $P \leq 0.05$ (unadjusted for multiplicity) was considered to be nominally statistically significant.

The minimally important difference (MID) was defined as the smallest difference in scores between groups in the scales of interest, which patients perceived as beneficial. Literature-based threshold values for MID were used for scales in the EORTC QLQ-C30 [21]. Because there are not any published MIDs on the QLQ-BR23, a 10-point change was considered consistent with previous estimates [22]. For functional scales, an increase in change score from baseline of $\geq 1$ MID was defined as "improved," a decrease of $\geq 1$ MID was defined as "worsened," and a change in either direction of $<1$ MID was defined as "stable." For symptom scales, the same criteria were applied with reverse direction. Proportions of patients classified as "improved," "stable," or "worsened" were calculated for each scale and cycle. Tests of proportions were done using Chi squared or Fisher's exact tests, as appropriate. Cox analysis was used 
Table 1 Baseline (a) patient characteristics and demographics, (b) health-related quality-of-life scores

\begin{tabular}{|c|c|c|c|}
\hline Parameter & Eribulin $(n=472)$ & Capecitabine $(n=444)$ & Total $(n=916)$ \\
\hline Age, median, years (SD; range) & $54.5(10.3 ; 24.0-80.0)$ & $53.0(10.3 ; 26.0-80.0)$ & $54.0(10.3 ; 24.0-80.0)$ \\
\hline \multicolumn{4}{|l|}{ Race, $n(\%)$} \\
\hline White & $424(89.8)$ & $402(90.5)$ & $826(90.2)$ \\
\hline Other & $48(10.2)$ & $42(9.5)$ & $90(9.8)$ \\
\hline Body mass index, median $\left(\mathrm{kg} / \mathrm{m}^{2}\right)(\mathrm{SD} ;$ range $)$ & $26.7(5.5 ; 12.7-62.1)$ & $28.2(5.8 ; 16.2-52.8)$ & $27.8(5.6 ; 12.7-62.1)$ \\
\hline \multicolumn{4}{|l|}{ ECOG PS, $n(\%)$} \\
\hline 0 & $228(48.3)$ & $195(43.9)$ & $423(46.2)$ \\
\hline 1 & $236(50.0)$ & $241(54.3)$ & $477(52.1)$ \\
\hline 2 & $8(1.7)$ & $8(1.8)$ & $16(1.7)$ \\
\hline \multicolumn{4}{|l|}{ HER2 status, $n(\%)$} \\
\hline Positive & $80(16.9)$ & $73(16.4)$ & $153(16.7)$ \\
\hline Negative & $309(65.5)$ & $301(67.8)$ & $610(66.6)$ \\
\hline Unknown & $83(17.6)$ & $70(15.8)$ & $153(16.7)$ \\
\hline \multicolumn{4}{|l|}{ Triple-negative disease, $n(\%)$} \\
\hline No & $349(73.9)$ & $343(77.3)$ & $692(75.5)$ \\
\hline Yes & $123(26.1)$ & $101(22.7)$ & $224(24.5)$ \\
\hline \multicolumn{4}{|c|}{ Number of prior chemotherapy regimens for metastatic disease, $n(\%)$} \\
\hline 1 & $130(27.5)$ & $122(27.5)$ & $252(27.5)$ \\
\hline 2 & $271(57.4)$ & $255(57.4)$ & $526(57.4)$ \\
\hline$\geq 3$ & $71(15.0)$ & $67(15.1)$ & $138(15.1)$ \\
\hline
\end{tabular}

(b)

\begin{tabular}{|c|c|c|}
\hline Domain & $\begin{array}{l}\text { Eribulin }^{\mathrm{a}} \\
(n=554)\end{array}$ & $\begin{array}{l}\text { Capecitabine }^{\mathrm{b}} \\
(n=548)\end{array}$ \\
\hline \multicolumn{3}{|l|}{ EORTC QLQ-C30 (mean [SD]) } \\
\hline GHS/QoL & $56.3(22.21)$ & $54.7(21.67)$ \\
\hline Physical functioning & $72.9(21.00)$ & $71.9(20.68)$ \\
\hline Role functioning & $73.4(27.68)$ & $70.0(29.27)$ \\
\hline Emotional functioning & $68.8(23.00)$ & $68.4(24.15)$ \\
\hline Cognitive functioning & $81.5(20.36)$ & $81.4(21.18)$ \\
\hline Social functioning & $75.4(26.28)$ & 73.4 (28.19) \\
\hline Fatigue & $37.4(23.70)$ & $38.0(24.72)$ \\
\hline Nausea and vomiting & $10.0(18.04)$ & $10.1(19.33)$ \\
\hline Pain & $31.8(28.41)$ & $32.9(29.45)$ \\
\hline Dyspnea & $23.3(27.56)$ & $25.1(29.45)$ \\
\hline Insomnia & $31.3(29.34)$ & $31.1(30.98)$ \\
\hline Appetite loss & $20.8(28.13)$ & $23.2(29.76)$ \\
\hline Constipation & $13.2(23.43)$ & $14.5(26.23)$ \\
\hline Diarrhea & $8.1(16.73)$ & $8.2(17.20)$ \\
\hline Financial difficulties & $32.6(33.83)$ & $30.1(32.62)$ \\
\hline \multicolumn{3}{|l|}{ EORTC QLQ-BR23 (mean [SD]) } \\
\hline Body image & $64.7(28.73)$ & $64.3(30.23)$ \\
\hline Sexual functioning & $14.0(20.34)$ & $16.5(22.51)$ \\
\hline Sexual enjoyment & $47.0(25.27)$ & $53.6(26.13)$ \\
\hline Future perspective & $32.1(31.29)$ & $31.0(30.84)$ \\
\hline Systemic therapy side-effects & $21.4(16.16)$ & $22.9(17.17)$ \\
\hline Breast symptoms & $19.2(22.74)$ & $20.3(24.86)$ \\
\hline
\end{tabular}


Table 1 continued

(b)

\begin{tabular}{lll}
\hline Domain & $\begin{array}{l}\text { Eribulin }^{\mathrm{a}} \\
(n=554)\end{array}$ & $\begin{array}{l}\text { Capecitabine }^{\mathrm{b}} \\
(n=548)\end{array}$ \\
\hline Arm symptoms & $25.1(26.28)$ & $26.4(26.25)$ \\
Upset by hair loss & $51.6(38.01)$ & $49.5(38.31)$ \\
\hline
\end{tabular}

Data shown are mean (SD). The italicized values represent symptom scales

BR23 breast cancer module (23 questions), ECOG PS Eastern Cooperative Oncology Group performance status, EORTC European Organisation for Research and Treatment of Cancer, GHS global health status, HER2 human endocrine receptor 2, $Q L Q$-C3O quality-of-life questionnaireCore 30 questions, $Q o L$ quality of life, $S D$ standard deviation

${ }^{\mathrm{a}} n=554(\mathrm{QLQ}-\mathrm{C} 30)$ or $536(\mathrm{BR} 23)$

${ }^{\mathrm{b}} n=548(\mathrm{QLQ}-\mathrm{C} 30)$ or $526(\mathrm{BR} 23)$

to compare the MID changes for eribulin versus capecitabine (using a reference HR of 1). Adjusted values are stated for the HR.

Time to symptom worsening (TSW) was defined as the time until clinically meaningful deterioration by a specified threshold for each patient-reported endpoint (such as, the MID values) was observed. TSW was calculated for each HRQoL scale using Kaplan-Meier curves. A proportional hazards model (censoring on death, study drop-out, or study discontinuation) was used to estimate adjusted HR values of TSW plus each respective $95 \%$ CI. For patients with $>1$ TSW event or who deteriorated without improvement, a generalized estimating equation was used to estimate the relative probabilities of observing TSW between treatment arms.

\section{Results}

\section{HRQoL population}

Of 1102 ITT patients randomized in study 301, 1062 (96.4\%) completed the EORTC questionnaire at baseline and thus formed the HRQoL population. The populations were broadly comparable between the treatment arms (Table 1a). The baseline scores for both questionnaires were similar (Table 1b). Across the symptom scales of the QLQC30, patients in both treatment arms had worse scores on fatigue, pain, insomnia, and financial difficulties (means >30). The scores on QLQ-C30 functional scales were generally good (mean values around and above 70) with the exception of GHS/QoL scale where mean scores around 50 suggest significant impact of disease [23]. However, the breast-cancer-specific functional scales of the QLQ-BR23 showed impact on all domains (mean scores 32-65), in particular, on sexual functioning (mean score 14.0; Table 1b).

Compliance for completing the EORTC questionnaires during the study was $\geq 85 \%$ until 12 months, but was lower at 18 and 24 months (73-83\%), and sample sizes decreased due to study attrition (Table 2). Due to smaller sample sizes, analyses after 6 months should be interpreted with caution.

\section{Treatment effects on symptoms}

Exposure to both treatments during the study was comparable between the two arms. Patients in the eribulin arm received a median of six treatment cycles, whereas patients in the capecitabine arm received a median of five treatment cycles. Overall, 177 patients (eribulin: $32.5 \%$, capecitabine: $32.4 \%$ ) in either arm underwent dose reduction. The most common reasons for dose reduction were neutropenia in the eribulin arm (22.6\%), and palmar-plantar erythrodysesthesia syndrome $(4.9 \%)$ in the capecitabine arm.

During the course of the study, patients receiving capecitabine had comparatively more-severe symptoms (that is, higher symptom scores) for nausea and vomiting $(P<0.001$; Fig. 1a and online resource Fig. S1; online resource Table $\mathrm{S} 1)$ and diarrhea $(P<0.001)$ compared with those treated with eribulin (Fig. 1a). The differences were clinically significant, as a higher proportion of patients who received capecitabine versus eribulin experienced clinically meaningful worsening of nausea and vomiting (MID 8; HR 1.177 [95 \% CI 1.013, 1.367]; $P<0.05$ ) and diarrhea (MID 7; HR 1.189 [95\% CI 1.020, 1.385]; $P<0.05$; Fig. 1b). Typically, the differences appeared to be greatest at 6 weeks, and declined thereafter.

In comparison, patients receiving eribulin had worse mean scores for the systemic therapy side-effects symptom scale (which included dry mouth, different tastes, irritated eyes, feeling ill, hot flushes, headaches, and hair loss; $P<0.001)$, and upset by hair loss $(P<0.05$; Fig. 1a). A higher proportion of patients treated with eribulin experienced clinically meaningful worsening of systemic therapy side-effects than those treated with capecitabine (MID 10; HR 0.821 [95 \% CI 0.707, 0.953]; $P<0.01$; Fig. 1b). 
Table 2 Proportion of patients completing questionnaires at scheduled visits

\begin{tabular}{lll}
\hline Visit & $\begin{array}{l}\text { Eribulin }(\%) \\
(n=554)\end{array}$ & $\begin{array}{l}\text { Capecitabine }(\%) \\
(n=548)\end{array}$ \\
\hline Baseline & $96.8(536 / 554)$ & $96.0(526 / 548)$ \\
6 weeks & $91.1(450 / 494)$ & $86.6(419 / 484)$ \\
3 months & $89.2(329 / 369)$ & $87.7(299 / 341)$ \\
6 months & $87.4(167 / 191)$ & $87.6(170 / 194)$ \\
12 months & $86.2(56 / 65)$ & $87.5(63 / 72)$ \\
18 months & $73.3(22 / 30)$ & $82.8(24 / 29)$ \\
24 months & $76.5(13 / 17)$ & $75.0(15 / 20)$ \\
\hline
\end{tabular}

Data show patients who completed at least 1 question in the European Organisation for Research and Treatment of Cancer questionnaire among those who completed a baseline questionnaire. \%, percent of all patients who were scheduled to complete a questionnaire at visit time (that is, patients who had not progressed or been censored)

The analysis of TSW supported the interpretation of the MID thresholds. Patients receiving capecitabine had significantly shorter TSW for nausea and vomiting (MID 8; 7.6 vs. 10.2 months; $P<0.05$ ), and diarrhea (MID 7; 8.4 vs. 11.5 months; $P<0.05)$ than those treated with eribulin. Similarly, patients treated with eribulin had significantly shorter TSW for systemic therapy side-effects (MID 10; 7.6 vs. 9.7 months; $P<0.05$; Fig. 1c) compared with those treated with capecitabine.

\section{Treatment effects on patient functioning}

In the longitudinal analyses, baseline HRQoL scores were significantly associated with the change in HRQoL across all EORTC scales $(P<0.001)$; that is, worse baseline scores were predictive of worse scores while on treatment. There were no differences between the two treatment arms in terms of impact on patients' functioning over time, as measured by changes in EORTC QLQ-C30 scores for functional scales (Fig. 2a). However, patients receiving eribulin had comparatively worse scores on the body image $(P<0.001)$ and sexual functioning scales $(P<0.05)$, measured by QLQ-BR23, than those receiving capecitabine (Fig. 2a).

As indicated by the MID analysis, $10-35 \%$ of patients in both treatment arms experienced a clinically significant worsening of their functioning, suggesting that the majority of patients experienced stable or improved functioning. No statistically significant differences over the course of the study were observed between the treatment groups, except that a higher proportion of patients receiving capecitabine reported a meaningful worsening on the future perspective scale than those receiving eribulin (MID 10; HR 1.173 [95\% CI 1.015, 1.356]; $P<0.05$; Fig. 2b).
Fig. 1 Effects of eribulin and capecitabine on physical symptom scales of the EORTC QLQ-C30 and QLQ-BR23 a differences in mean scores; b proportion of patients with worsened symptoms; c differences in median time to symptom worsening

In the ITT population, median TSW was similar for the majority of the EORTC functional scales and the GHS/QoL scale, with only 1-2 months' difference between the treatment arms. Patients receiving eribulin had significantly longer TSW for body image (MID 10; 8.9 vs. 6.0 months; $P<0.05$ ) and future perspective (MID 10; 6.1 vs. 4.7 months; $P<0.05$; Fig. 2c) than those treated with capecitabine.

\section{Treatment effects in patient subgroups by receptor status}

Overall, the results in the HER2-negative and triple-negative subgroups were similar to those in the overall population in all analyses (data not shown). However, in patients with triple-negative disease, significant differences were observed in the TSW analyses. Importantly, TSW in overall GHS/QoL was significantly longer in patients treated with eribulin than those treated with capecitabine (median time 6.2 vs. 6.0 months; $P<0.01$; Fig. 3). This difference in median TSW may not appear clinically meaningful, however, a separation of the Kaplan-Meier survival curves is observed beyond 6 months, which is likely to explain the statistically significant difference between the two treatments. The median TSWs were also longer in the eribulin arm compared with capecitabine arm for fatigue ( 8.9 vs. 6.1 months; $P<0.01$ ), nausea and vomiting (9.9 vs. 6.5 months; $P<0.05$ ), pain (8.1 vs. 5.4 months; $P<0.05$ ), and diarrhea (11.6 vs. 6.6 months; $P<0.01$ ); as well as for the functional scales of body image (6.7 vs. 6.0 months; $P<0.05)$ and future perspective (6.0 vs. 4.8 months; $P=0.01$ ). The TSW for systemic therapy side-effects appeared shorter for patients treated with eribulin than those receiving capecitabine, but this difference was not statistically significant $(4.9$ vs. 7.2 months; $P>0.05$ ).

\section{Discussion}

In this phase 3 trial comparing eribulin with capecitabine in patients with locally advanced or MBC previously treated with an anthracycline and a taxane, significant differences in physical symptoms/side-effects were observed, reflecting the different toxicity profiles of the drugs. Patients treated with capecitabine had worse scores, and more rapid TSW for gastrointestinal symptoms (nausea and vomiting, diarrhea), whereas patients treated with eribulin had worse 
(a) HRQoL symptom differences on the C30 questionnaire

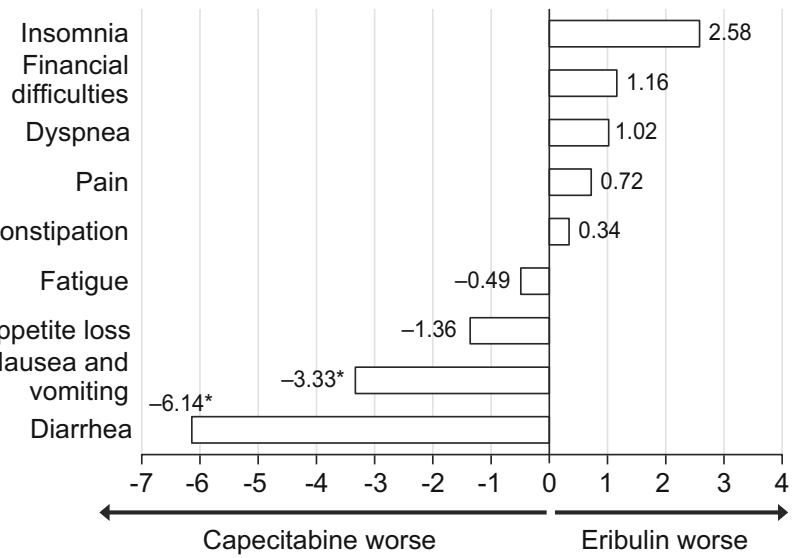

HRQoL symptom differences on the BR23 questionnaire

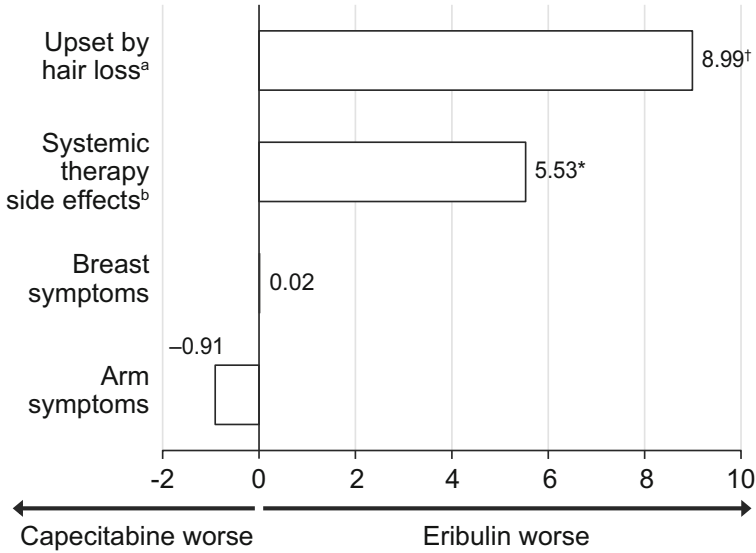

${ }^{*} P<0.001 ;{ }^{\dagger} P<0.05$

a Upset by hair loss is only answered by patients who experience hair loss. 'systemic therapy side effects include dry mouth, different tastes, irritated eyes, feel ill, hot flushes, headaches, hair loss. The linear mixed model estimated the change from baseline through month 24 . Model adjusted with the following covariates: baseline patient-reported outcomes, age, human endocrine receptor 2 status, triple-negative status, European Cooperative Oncology Group score, number of prior chemotherapy regimens, hormone status, number of organs involved, and visceral involvement. EORTC, European Organisation for Research and Treatment of Cancer; HRQoL, health-related quality of life; QLQ-C30/BR23, Quality-of-life questionnaire Core 30 questions/Breast module.

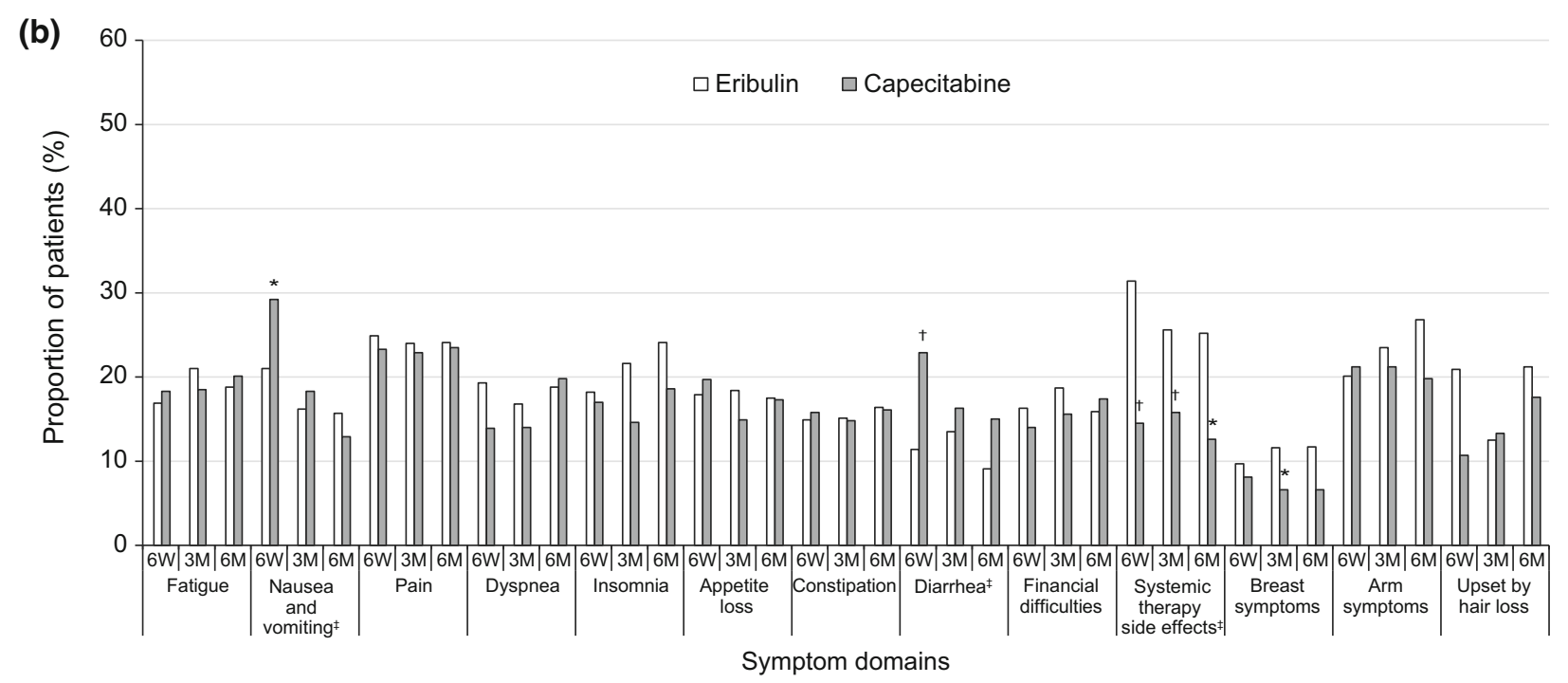

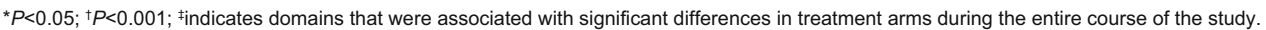

Minimum important differences used in the analyses: fatigue $=13$, nausea and vomiting $=8$, pain $=13$, dyspnea $=9$, insomnia $=13$, appetite loss $=14$, constipation $=13$, diarrhea $=7$, Minimum important differences used in the analyses: fatigue $=13$, nausea and vomiting $=8$, pain $=13$, dyspnea $=9$, insomnia $=13$, appetite loss $=14$,
financial difficulties $=10$, systemic therapy side effects $=10$, breast symptoms $=10$, arm symptoms $=10$, upset by hair loss $=10 . \mathrm{W}$, weeks; $\mathrm{M}$, months.

(c)

Systemic therapy side effects

Upset by hair loss

Dyspnea

Insomnia

Breast symptoms

Arm symptoms

Constipation

Fatigue

Financial difficulties

Appetite loss

Pain

Nausea and vomiting

Diarrhea

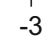

3
$-2.04^{*}$

$$
-1.74
$$

$-0.95$

$-0.95$

$-0.36$

$-0.29$

$-0.26$

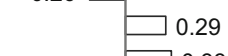

$\square .29$

0.56

1.12

$2.53^{\star}$

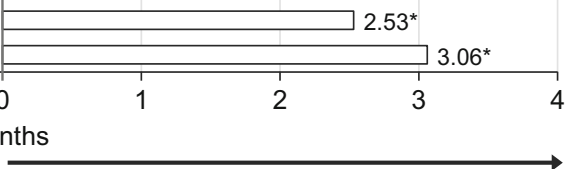

${ }^{*} P<0.05$

Capecitabine worse

Eribulin worse

The adjusted covariates include age group, race group, and categorical Eastern Cooperative Oncology Group score. 
(a)

HRQoL profile differences on the C30 questionnaire

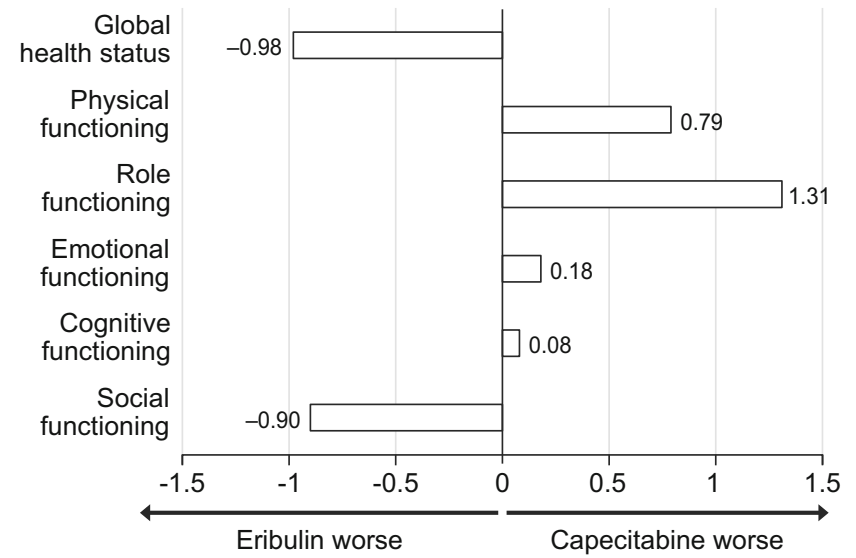

HRQoL profile differences on the BR23 questionnaire

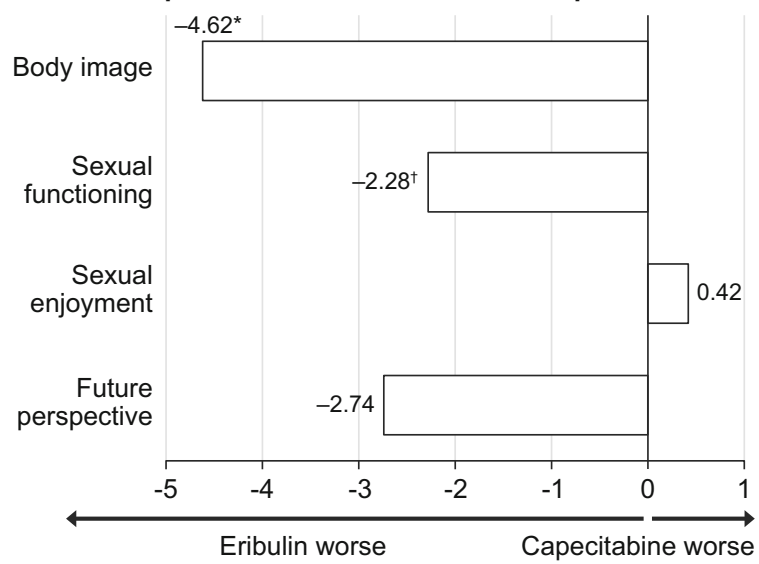

${ }^{*} P<0.001 ;{ }^{\dagger} P<0.05$

The linear mixed model estimated the change from baseline through month 24. Model adjusted with the following covariates: baseline patient-reported outcomes, age, human endocrine receptor 2 status, triple-negative status, European Cooperative Oncology Group score, number of prior chemotherapy regimens, hormone status, number of organs involved, and visceral involvement. EORTC, European Organisation for Research and Treatment of Cancer; HRQoL, health-related quality of life; QLQ-C30/BR23, Quality-of-life questionnaire Core 30 questions/Breast module.

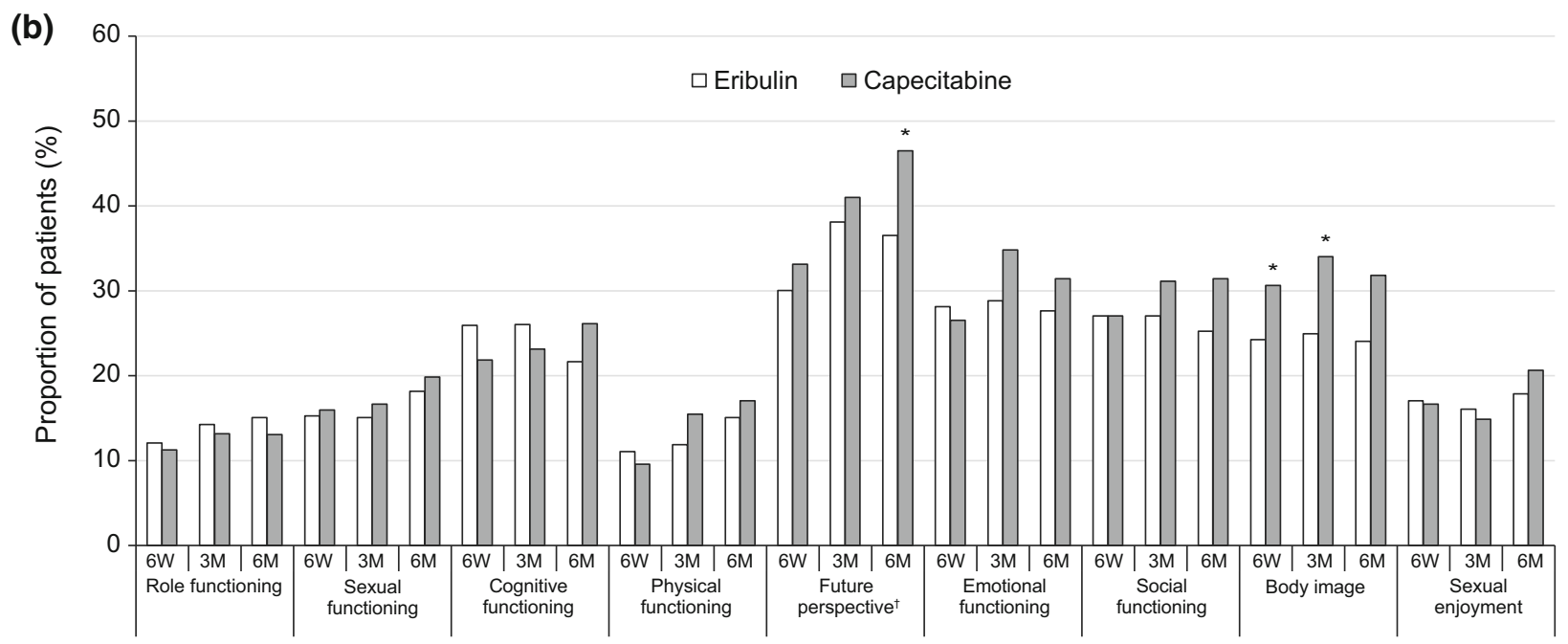

Function domains

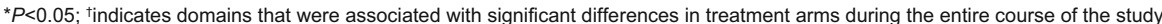

Minimum important differences used in the analyses: physical functioning $=14$, role functioning $=19$, emotional functioning $=11$, cognitive functioning $=9$, social functioning $=11$, body image $=10$, sexual functioning $=10$, sexual enjoyment $=10$, future perspective $=10$. W, weeks; $M$, months.

(c)

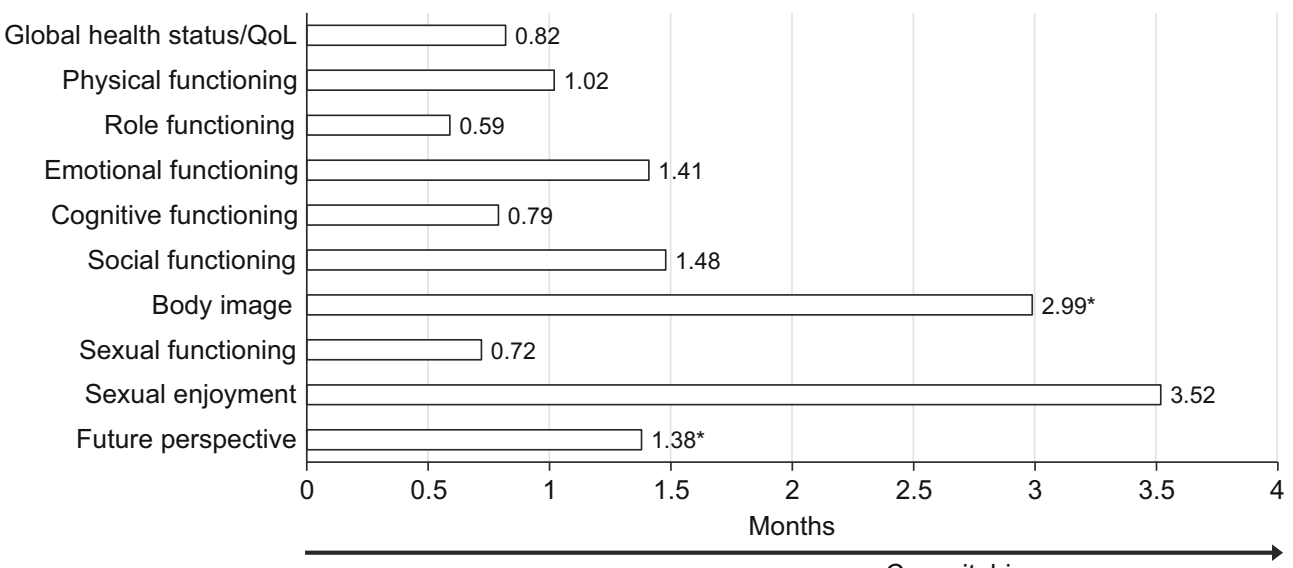

$P<0.05$

Capecitabine worse

The adjusted covariates include age group, race group, and categorical Eastern Cooperative Oncology Group score. 
4Fig. 2 Effects of eribulin and capecitabine on function scales of the EORTC QLQ-C30 and QLQ-BR23. a differences in mean scores; b proportion of patients with worsened symptoms; c differences in median time to symptom worsening

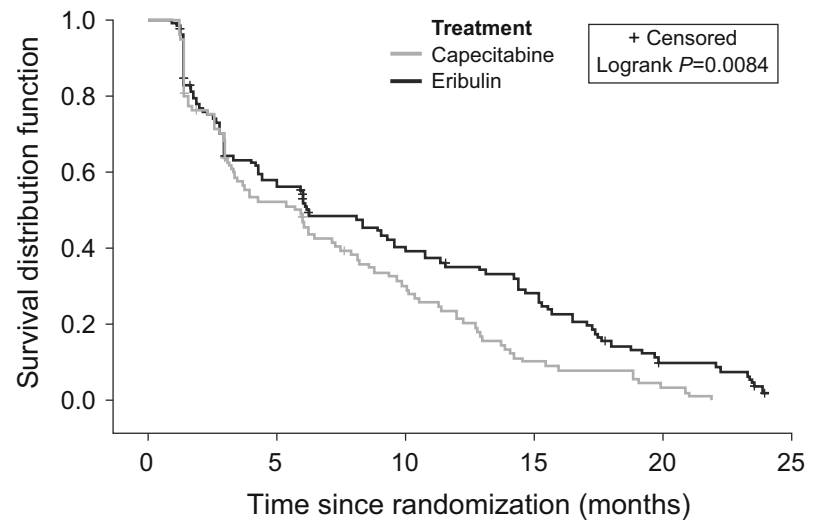

$\begin{array}{lcccccc}\text { Capecitabine } & 98 & 49 & 27 & 9 & 3 & 0 \\ \text { Eribulin } & 118 & 61 & 39 & 27 & 8 & 0\end{array}$

EORTC QLQ-C30, European Organisation for Research and Treatment of Cancer Quality-of-life Questionnaire-Core 30 questions.

Fig. 3 Effects of eribulin and capecitabine, in terms of time to symptom worsening, on overall global health status/quality-of-life scale of the EORTC QLQ-C30 in patients with triple-negative disease

scores for systemic therapy side-effects (dry mouth, food and drink taste, eyes painful, hair loss, feeling ill/unwell, hot flushes, headaches). These results were not only statistically significant in the longitudinal models but were also clinically meaningful, as measured by the MID analyses and TSW. Typically, the differences appeared to be greatest at 6 weeks, and declined thereafter. This is in alignment with the literature which suggests that during the course of their disease, over $50 \%$ of patients will experience nausea and vomiting, with a large proportion experiencing these within the first week of treatment $[10,24]$.

Despite the above side-effects, the majority of patients $(65-90 \%)$ in both treatment groups maintained or improved their functioning relative to baseline. Based on the group-level data over time using the pattern-mixture model, patients treated with eribulin had worse body image scores than those receiving capecitabine. While this finding may seem contradictory to the TSW results, which show that patients treated with eribulin compared with capecitabine have longer TSW for body image, this can be explained by the nature of the two different approaches. TSW is an analytic approach that censors data on the time to meaningful decline by a predefined threshold, whereas, the longitudinal evaluation of the raw score change is not censored. Therefore, while patients may have worse scores for these domains in the eribulin arm, we observe that there scores do not decline more rapidly compared to capecitabine treatment to a point of meaningful worsening.
Notably, in patients with triple-negative disease, eribulin also demonstrated a significant delay in time to symptom worsening of overall GHS/QoL, as well as fatigue, nausea and vomiting, diarrhea, and pain when compared with capecitabine. Although the 0.2-month improvement in median TSW of overall GHS/QoL with eribulin compared with capecitabine may not translate to an early clinical benefit, a larger separation of the curves is observed beyond 6 months, potentially due to a subset of patients responding well to treatment.

Capecitabine is widely considered to be a chemotherapy drug with manageable toxicity and a favorable risk:benefit profile in patients with MBC $[25,26]$. It is often used as a first-line treatment in older and frail patients to achieve disease control without significant side-effects [25, 27]. The capecitabine dose used in this study $\left(1.25 \mathrm{~g} / \mathrm{m}^{2}\right.$ orally twice-daily on days 1-14 per 21-day cycle) is approved by the United States Food and Drug Administration, and has been used in other clinical trials in patients with MBC [2830]. A lower dose of capecitabine, typically $1000 \mathrm{mg} / \mathrm{m}^{2}$ twice-daily for 14 days per 21-day cycle, has also been investigated with similar efficacy but reportedly better tolerability [28]. In our study, treatment exposure to capecitabine was comparable to eribulin, with similar proportions of patients in both arms experiencing dose reduction. The observation that the impact of eribulin on patient functioning and HRQoL, despite worse systemic side-effect scores, is similar to that of capecitabine, is therefore noteworthy, especially from a clinical perspective.

HRQoL is increasingly being recognized as a valuable endpoint of cancer care by payers, regulators, prescribers, and patients. Patient-reported symptom endpoints have prognostic value in clinical trials and support the importance of assessing the patient's views in the development of new therapies [31]. Therefore, while the development of new cancer therapies should clearly focus on improving efficacy, ideally in terms of OS, maximizing HRQoL is also an important goal, particularly in the setting of advanced disease. Where treatments have comparable tumor-related outcomes, but competing toxicity profiles or differing logistics of administration, HRQoL measurement may help patients and clinicians to guide treatment decisions [32]. The MID assessment described here was defined as the smallest difference in scores between groups in the scales of interest, which patients perceived as beneficial. Therefore, by definition, MID-based improvements are indicative of treatment benefit, and could guide physicians in making treatment decisions.

The measurement of HRQoL and interpretation of HRQoL outcomes are, however, challenging. A review of clinical trials in patients with MBC that included measurement of HRQoL concluded that this assessment does 
not always provide additional information that is not already clear from other clinical outcomes, such as toxicity [32]. Indeed, in the current study, HRQoL changes in individual symptom scales of the EORTC QLQ-C30 and QLQ-BR23 instruments detected differences on patient reporting corresponding to the known adverse event profile for each agent. Nevertheless, no differences between the two treatments were observed for functional and overall HRQoL outcomes. HRQoL instruments may, however, lack sufficient precision to detect differences between treatments among a relatively homogeneous clinical trial population [33]. Patients may also find it easier to rate individual symptom scales accurately than a global HRQoL scale [33].

Chemotherapy-induced gastrointestinal symptoms (nausea, vomiting, and diarrhea) are common side-effects that can be debilitating for patients $[10,11]$. Importantly, diarrhea can interfere with cancer treatment by forcing dose delays or reductions [34]. Diarrhea is one of the principal dose-limiting toxicities of capecitabine and this is reflected in the study findings, with eribulin being associated with comparatively lower symptom scores for diarrhea as well as a lower proportion of patients reporting worsening diarrhea, and a longer TSW for this symptom. Patients on capecitabine reported worse nausea/vomiting than those on eribulin, whereas the scale scores for other systemic therapy side-effects were significantly worse with eribulin. Together, this information will contribute to discussions with patients during the treatment decision-making process. Review of the observed toxicity experience of patients in this study (any grade adverse event) were consistent with the patient-reported symptom experience reported in this study: patients on capecitabine experienced more adverse events of diarrhea (29 vs. $14 \%$ for eribulin), vomiting (17 vs. $12 \%$ for eribulin), anorexia (15 vs. $13 \%$ for eribulin) and nausea (24 vs. $22 \%$ for eribulin) whereas patients on eribulin experienced more adverse events of alopecia (35 vs. $18 \%$ for capecitabine) and fatigue (17 vs. $15 \%$ for capecitabine) [35].

This HRQoL analysis has several strengths. It is based on data from a large-scale, randomized, clinical trial, using 2 well-validated instruments for data collection-the EORTC QLQ-C30 instrument and its breast-cancer-specific supplementary module, QLQ-BR23. Compliance was good throughout most of the study ( $\geq 80 \%$ to the 12 -month time point). Content validity for the symptom scales used has been validated in patients with advanced breast cancer or MBC in a phase 2 study of eribulin (E7389-G000-211; ClinicalTrials.gov identifier: NCT00246090) [36]. However, the analyses may be limited by the sample size for QoL analysis that decreased sharply from the 12-month time point due to death or discontinuation from the parent study, which is frequently observed in the metastatic setting due to the natural history of the disease. It should also be noted that the assessment instruments used for the study do not specifically capture the symptoms particularly related to capecitabine (namely, "hand-foot syndrome") and eribulin (e.g., peripheral neuropathy).

In conclusion, the majority of patients with pretreated locally advanced or MBC who were treated with either eribulin or capecitabine did not experience an overall deterioration in functioning or GHS/QoL. Eribulin and capecitabine had similar effects on patients' HRQoL scores reflecting the known side-effect profiles of these two agents. Along with the efficacy and toxicity findings of study 301 [12] and study 305/EMBRACE [11], the HRQoL results from this study 301 analyses may help patients with MBC and their oncologists to make more informed treatment decisions.

Acknowledgments Editorial assistance was provided by Oxford PharmaGenesis and Virgo Health. Education and was supported by Eisai Inc.

Funding This study was funded by Eisai Inc.

\section{Compliance with ethical standards}

Disclosures JC has received consultancy fees from Roche and Celgene, and honoraria from Roche, Celgene, Novartis, and Eisai. SH has received fees for planning and execution of the QoL analysis and report generation, but no financial support for manuscript development. CT has received honoraria from Roche, Eisai, Astra Zeneca, and Pfizer. EP has no relevant conflicts of interest to disclose. AA participated in advisory boards organized by Eisai, Roche, and Bayer. LY: Research funding to the Institution from Nektar Therapeutics, Boehringer Ingelheim, Roche-Hoffman, Eisai Inc., Genentech, MedImmune, and Puma Biotechnology inc. SM is a former employee of Eisai. AF is an Eisai employee. PK has received consultancy fees from Eisai and Celgene. GV has received honoraria from Eisai, Roche, and Novartis.

Ethical Approval The primary study was approved by the appropriate institutional and/or national ethics committee. All procedures performed in studies involving human participants were in accordance with the ethical standards of the institutional and/or national research committee and with the 1964 Helsinki declaration and its later amendments or comparable ethical standards. Written informed consent was obtained from all individual participants included in the primary study.

Open Access This article is distributed under the terms of the Creative Commons Attribution-NonCommercial 4.0 International License (http://creativecommons.org/licenses/by-nc/4.0/), which permits any noncommercial use, distribution, and reproduction in any medium, provided you give appropriate credit to the original author(s) and the source, provide a link to the Creative Commons license, and indicate if changes were made.

\section{References}

1. Greenberg PA, Hortobagyi GN, Smith TL, Ziegler LD, Frye DK, Buzdar AU (1996) Long-term follow-up of patients with 
complete remission following combination chemotherapy for metastatic breast cancer. J Clin Oncol 14:2197-2205

2. Belfiglio M, Fanizza C, Tinari N, Ficorella C, Iacobelli S, Natoli C, Consorzio Interuniversitario Nazionale per la Bio-Oncologia (CINBO) (2012) Meta-analysis of phase III trials of docetaxel alone or in combination with chemotherapy in metastatic breast cancer. J Cancer Res Clin Oncol 138:221-229. doi:10.1007/ s00432-011-1091-0

3. Park IH, Lee KS, Ro J (2015) Effects of second and subsequent lines of chemotherapy for metastatic breast cancer. Clin Breast Cancer 15:e55-e62. doi:10.1016/j.clbc.2014.09.001

4. Jones SE (2008) Metastatic breast cancer: the treatment challenge. Clin Breast Cancer 8:224-233. doi:10.3816/CBC.2008.n. 025

5. Di Maio M, Gallo C, Leighl NB, Piccirillo MC, Daniele G, Nuzzo F, Gridelli C, Gebbia V, Ciardiello F, De Placido S, Ceribelli A, Favaretto AG, de Matteis A, Feld R, Butts C, Bryce J, Signoriello S, Morabito A, Rocco G, Perrone F (2015) Symptomatic toxicities experienced during anticancer treatment: agreement between patient and physician reporting in three randomized trials. J Clin Oncol 33:910-915. doi:10.1200/JCO.2014. 57.9334

6. Basch E (2010) The missing voice of patients in drug-safety reporting. NEngl J Med 362:865-869. doi:10.1056/NEJMp0911494

7. Basch E, Reeve BB, Mitchell SA, Clauser SB, Minasian LM, Dueck AC, Mendoza TR, Hay J, Atkinson TM, Abernethy AP, Bruner DW, Cleeland CS, Sloan JA, Chilukuri R, Baumgartner P, Denicoff A, St Germain D, O’Mara AM, Chen A, Kelaghan J, Bennett AV, Sit L, Rogak L, Barz A, Paul DB, Schrag D (2014) Development of the National Cancer Institute's patient-reported outcomes version of the common terminology criteria for adverse events (PRO-CTCAE). J Natl Cancer Inst 106:pii: dju244. doi: 210.1093/jnci/dju1244. doi:10.1093/jnci/dju244

8. Brettschneider C, Lühmann D, Raspe H (2011) Informative value of Patient Reported Outcomes (PRO) in Health Technology Assessment (HTA). GMS Health Technol Assess 7:Doc01. doi: $10.3205 /$ hta000092

9. Reed E, Simmonds P, Haviland J, Corner J (2012) Quality of life and experience of care in women with metastatic breast cancer: a cross-sectional survey. J Pain Symptom Manage 43:747-758. doi:10.1016/j.jpainsymman.2011.05.005

10. Ballatori E, Roila F (2003) Impact of nausea and vomiting on quality of life in cancer patients during chemotherapy. Health Qual Life Outcomes 1:46. doi:10.1186/1477-7525-1-46

11. Cortes J, O'Shaughnessy J, Loesch D, Blum JL, Vahdat LT, Petrakova K, Chollet P, Manikas A, Diéras V, Delozier T, Vladimirov V, Cardoso F, Koh H, Bougnoux P, Dutcus CE, Seegobin S, Mir D, Meneses N, Wanders J, Twelves C, EMBRACE investigators (2011) Eribulin monotherapy versus treatment of physician's choice in patients with metastatic breast cancer (EMBRACE): a phase 3 open-label randomised study. Lancet 377:914-923. doi:10.1016/S0140-6736(11)60070-6

12. Kaufman PA, Awada A, Twelves C, Yelle L, Perez EA, Velikova G, Olivo MS, He Y, Dutcus CE, Cortes J (2015) Phase III openlabel randomized study of eribulin mesylate versus capecitabine in patients with locally advanced or metastatic breast cancer previously treated with an anthracycline and a taxane. J Clin Oncol 33:594-601. doi:10.1200/JCO.2013.52.4892

13. Halaven (eribulin mesylate) injection [prescribing information] (2014) Eisai Inc., Woodcliff Lake, NJ, USA

14. Halaven $0.44 \mathrm{mg} / \mathrm{ml}$ solution for injection [summary of product characteristics] Eisai Europe Limited, Hertfordshire, England, UK

15. Wilson IB, Cleary PD (1995) Linking clinical variables with health-related quality of life. A conceptual model of patient outcomes. JAMA 273:59-65
16. Aaronson NK, Ahmedzai S, Bergman B, Bullinger M, Cull A, Duez NJ, Filiberti A, Flechtner H, Fleishman SB, de Haes JC, Kaasa S, Klee M, Osoba D, Razavi D, Rofe PB, Schraub S, Sneeuw K, Sullivan M (1993) The European Organization for Research and Treatment of Cancer QLQ-C30: a quality-of-life instrument for use in international clinical trials in oncology. J Natl Cancer Inst 85:365-376

17. Osoba D, Zee B, Pater J, Warr D, Kaizer L, Latreille J (1994) Psychometric properties and responsiveness of the EORTC quality of Life Questionnaire (QLQ-C30) in patients with breast, ovarian and lung cancer. Qual Life Res 3:353-364

18. Sprangers MA, Groenvold M, Arraras JI, Franklin J, te Velde A, Muller M, Franzini L, Williams A, de Haes HC, Hopwood P, Cull A, Aaronson NK (1996) The European Organization for research and treatment of cancer breast cancer-specific quality-of-life questionnaire module: first results from a three-country field study. J Clin Oncol 14:2756-2768

19. EORTC Manual (2001) http://www.eortc.be/qol/files/SCMa nualQLQ-C30.pdf. Accessed June 3, 2015

20. Little RJ, Rubin DB (2002) Statistical analysis with missing data, 2nd edn. Wiley, New York

21. Cocks K, King MT, Velikova G, Martyn St-James M, Fayers PM, Brown JM (2011) Evidence-based guidelines for determination of sample size and interpretation of the European Organisation for the Research and Treatment of Cancer Quality of Life Questionnaire Core 30. J Clin Oncol 29:89-96. doi:10.1200/JCO.2010. 28.0107

22. Osoba D, Slamon DJ, Burchmore M, Murphy M (2002) Effects on quality of life of combined trastuzumab and chemotherapy in women with metastatic breast cancer. J Clin Oncol 20:3106-3113

23. Harrison CN, Mesa RA, Kiladjian JJ, Al-Ali HK, Gisslinger H, Knoops L, Squier M, Sirulnik A, Mendelson E, Zhou X, CopleyMerriman C, Hunter DS, Levy RS, Cervantes F, Passamonti F, Barbui T, Barosi G, Vannucchi AM (2013) Health-related quality of life and symptoms in patients with myelofibrosis treated with ruxolitinib versus best available therapy. $\mathrm{Br} \mathrm{J}$ Haematol 162:229-239. doi:10.1111/bjh.12375

24. Warr DG (2008) Chemotherapy-and cancer-related nausea and vomiting. Curr Oncol 15(Suppl 1):S4-S9

25. Ershler WB (2006) Capecitabine monotherapy: safe and effective treatment for metastatic breast cancer. Oncologist 11:325-335. doi:10.1634/theoncologist.11-4-325

26. Müller V, Fuxius S, Steffens CC, Lerchenmüller C, Luhn B, Vehling-Kaiser U, Hurst U, Hahn LJ, Soeling U, Wohlfarth T, Zaiss M (2014) Quality of life under capecitabine (Xeloda ${ }^{\circledR}$ ) in patients with metastatic breast cancer: data from a german noninterventional surveillance study. Oncol Res Treat 37:748-755. doi:10.1159/000369487

27. Gelmon K, Chan A, Harbeck N (2006) The role of capecitabine in first-line treatment for patients with metastatic breast cancer. Oncologist 11(Suppl 1):42-51. doi:10.1634/theoncologist.1190001-42

28. O'Shaughnessy JA, Kaufmann M, Siedentopf F, Dalivoust P, Debled M, Robert NJ, Harbeck N (2012) Capecitabine monotherapy: review of studies in first-line HER-2-negative metastatic breast cancer. Oncologist 17:476-484. doi:10.1634/ theoncologist.2011-0281

29. Sparano JA, Vrdoljak E, Rixe O, Xu B, Manikhas A, Medina C, Da Costa SC, Ro J, Rubio G, Rondinon M, Perez Manga G, Peck R, Poulart V, Conte P (2010) Randomized phase III trial of ixabepilone plus capecitabine versus capecitabine in patients with metastatic breast cancer previously treated with an anthracycline and a taxane. J Clin Oncol 28:3256-3263. doi:10.1200/JCO.2009. 24.4244

30. Buzdar AU, Xu B, Digumarti R, Goedhals L, Hu X, Semiglazov V, Cheporov S, Gotovkin E, Hoersch S, Rittweger K, Miles DW, 
O’Shaughnessy J, Tjulandin S, NO16853 trial group (2012) Randomized phase II non-inferiority study (NO16853) of two different doses of capecitabine in combination with docetaxel for locally advanced/metastatic breast cancer. Ann Oncol 23:589-597. doi:10.1093/annonc/mdr256

31. Osoba D (2011) Health-related quality of life and cancer clinical trials. Ther Adv Med Oncol 3:57-71. doi:10.1177/1758834010 395342

32. Goodwin PJ, Black JT, Bordeleau LJ, Ganz PA (2003) Healthrelated quality-of-life measurement in randomized clinical trials in breast cancer-taking stock. J Natl Cancer Inst 95:263-281

33. Ganz PA (2011) Assessing the quality and value of quality-of-life measurement in breast cancer clinical trials. J Natl Cancer Inst 103:196-199. doi:10.1093/jnci/djq542
34. Stein A, Voigt W, Jordan K (2010) Chemotherapy-induced diarrhea: pathophysiology, frequency and guideline-based management. Ther Adv Med Oncol 2:51-63. doi:10.1177/ 1758834009355164

35. Hudgens S, Briggs A, Velikova G, Forsythe A, McCutcheon S, Kind P (2014) Impact of treatment with eribulin (ERI) or capecitabine (CAP) for metastatic breast cancer (MBC) on EQ-5D utility derived from EORTC QLQ-C30 [abstract]. Ann Oncol 25(Suppl 4):iv357-iv360. Abstract 1046P

36. Simons WR (2013) Content validity of signs and symptoms measures with the EORTC core module in women with locally advanced or metastatic breast cancer (MBC): a phase II trial with eribulin mesylate [abstract]. J Clin Oncol 31 (suppl):abstract e17576 